\title{
Positive solution for a fractional singular boundary value problem with $p$-Laplacian operator
}

\author{
Fengli Yan, Mingyue Zuo and Xinan Hao*
}

\section{"Correspondence:}

haoxinan2004@163.com School of Mathematical Sciences, Qufu Normal University, Qufu, P.R. China

\section{照 Springer}

\begin{abstract}
In this paper, we consider a fractional singular three-point boundary value problem with $p$-Laplacian operator. The nonlinearity $f(t, u)$ may be singular at $t=0,1$ and $u=0$. Some properties of the associated Green function are obtained. By using the upper and lower solutions method and a fixed point theorem, the existence result of positive solution is established.
\end{abstract}

Keywords: Positive solution; Fractional singular BVP; $p$-Laplacian operator; Upper and lower solutions method

\section{Introduction}

In this paper, we investigate the following fractional three-point boundary value problem (BVP) with $p$-Laplacian operator:

$$
\left\{\begin{array}{l}
-D_{0^{+}}^{\alpha}\left(\varphi_{p}\left(D_{0^{+}}^{\beta} u(t)\right)\right)=f(t, u(t)), \quad 0<t<1, \\
u(0)=u(1)=u^{\prime}(0)=u^{\prime}(1)=0, \quad D_{0^{+}}^{\beta} u(0)=0, \quad D_{0^{+}}^{\beta} u(1)=b D_{0^{+}}^{\beta} u(\eta),
\end{array}\right.
$$

where $\alpha \in(1,2], \beta \in(3,4], D_{0^{+}}^{\alpha}$ and $D_{0^{+}}^{\beta}$ are the standard Riemann-Liouville derivatives, $\varphi_{p}(s)=|s|^{p-2} s, p>1, \varphi_{p}^{-1}=\varphi_{q}, \frac{1}{p}+\frac{1}{q}=1, \eta \in(0,1), b \in\left(0, \eta^{\frac{1-\alpha}{p-1}}\right), f(t, u):(0,1) \times(0,+\infty) \rightarrow$ $[0,+\infty)$ is continuous and may be singular at $t=0,1$ and $u=0$.

The differential equations with $p$-Laplacian operator have deep background in physics. In recent years, boundary value problems of fractional differential equations with or without $p$-Laplacian operator have been widely studied. By means of nonlinear analysis theory and methods, many existence and multiplicity results of solutions or positive solutions have been obtained, see [1-29] and the references therein.

In [11], Xu and Dong considered three-point BVP (1.1), but their nonlinearity $f:[0,1] \times$ $[0,+\infty) \rightarrow[0,+\infty)$ is continuous, the existence and uniqueness of positive solutions were obtained by using the upper and lower solutions method and Schauder's fixed point theorem.

By means of the lower and upper solutions method and monotone iterative technique, Liu et al. [12] investigated the existence of positive solutions for mixed fractional BVP with

(c) The Author(s) 2018. This article is distributed under the terms of the Creative Commons Attribution 4.0 International License (http://creativecommons.org/licenses/by/4.0/), which permits unrestricted use, distribution, and reproduction in any medium, provided you give appropriate credit to the original author(s) and the source, provide a link to the Creative Commons license, and indicate if changes were made. 
$p$-Laplacian operator

$$
\begin{cases}D_{0^{+}}^{\alpha}\left(\varphi_{p}\left({ }^{c} D_{0^{+}}^{\beta} u(t)\right)\right)=f\left(t, u(t),{ }^{c} D_{0^{+}}^{\beta} u(t)\right), & 0<t<1, \\ { }^{c} D_{0^{+}}^{\beta} u(0)=u^{\prime}(0)=0, \quad u(1)=r_{1} u(\eta), & { }^{c} D_{0^{+}}^{\beta} u(1)=r_{2}^{c} D_{0^{+}}^{\beta} u(\xi),\end{cases}
$$

where $\alpha, \beta \in(1,2], D_{0^{+}}^{\alpha}$ and ${ }^{c} D_{0^{+}}^{\beta}$ are the Riemann-Liouville fractional derivative and Caputo fractional derivative, respectively.

By using upper and lower solutions method, Wang and Xiang [13] established existence results of positive solution for a fractional BVP with $p$-Laplacian operator

$$
\begin{cases}D_{0^{+}}^{\alpha}\left(\varphi_{p}\left(D_{0^{+}}^{\beta} u(t)\right)\right)=f(t, u(t)), & 0<t<1, \\ u(0)=0, \quad u(1)=a u(\xi), & D_{0^{+}}^{\beta} u(0)=0,\end{cases}
$$

where $\alpha, \beta \in(1,2], a, b \in(0,1], \xi, \eta \in(0,1), D_{0^{+}}^{\alpha}$ and $D_{0^{+}}^{\beta}$ are the Riemann-Liouville fractional derivatives.

In [17], Zhang et al. studied the integral BVP of fractional differential equations with parameter and $p$-Laplacian operator

$$
\left\{\begin{array}{l}
-D_{0^{+}}^{\alpha}\left(\varphi_{p}\left(D_{0^{+}}^{\beta} u(t)\right)\right)=\lambda f(t, u(t), \quad 0<t<1, \\
u(0)=0, \quad D_{0^{+}}^{\beta} u(0)=0, \quad u(1)=\int_{0}^{1} u(s) d A(s),
\end{array}\right.
$$

where $\alpha \in(0,1], \beta \in(1,2], D_{0^{+}}^{\alpha}$ and $D_{0^{+}}^{\beta}$ are the Riemann-Liouville fractional derivatives, $\int_{0}^{1} u(s) d A(s)$ is the Riemann-Stieltjes integral, $f(t, u):(0,1) \times(0,+\infty) \rightarrow[0,+\infty)$ is continuous.

Motivated by the papers mentioned above, in this paper, we study the $p$-Laplacian fractional differential equation three-point BVP (1.1). The existence of positive solution is obtained by using the upper and lower solutions method and a fixed point theorem. It is worth pointing out that $f(t, u)$ may be singular at $t=0,1$ and $u=0$.

\section{Preliminaries and lemmas}

Let $\varphi_{p}\left(D_{0^{+}}^{\beta} u(t)\right)=v(t)$, then $v(0)=0, v(1)=b^{p-1} v(\eta)$. We now consider the following BVP:

$$
\left\{\begin{array}{l}
-D_{0^{+}}^{\alpha} \nu(t)=y(t), \quad 0<t<1 \\
v(0)=0, \quad v(1)=b^{p-1} v(\eta)
\end{array}\right.
$$

Lemma 2.1 ([11]) If $y \in C[0,1]$, then $B V P(2.1)$ has a unique solution

$$
v(t)=\int_{0}^{1} H(t, s) y(s) d s,
$$

where

$$
\begin{aligned}
& H(t, s)=h(t, s)+\frac{b^{p-1} t^{\alpha-1}}{1-b^{p-1} \eta^{\alpha-1}} h(\eta, s), \\
& h(t, s)=\frac{1}{\Gamma(\alpha)} \begin{cases}t^{\alpha-1}(1-s)^{\alpha-1}, & 0 \leq t \leq s \leq 1, \\
t^{\alpha-1}(1-s)^{\alpha-1}-(t-s)^{\alpha-1}, & 0 \leq s \leq t \leq 1 .\end{cases}
\end{aligned}
$$


From the above analysis, the BVP

$$
\left\{\begin{array}{l}
-D_{0^{+}}^{\alpha}\left(\varphi_{p}\left(D_{0^{+}}^{\beta} u(t)\right)\right)=y(t), \quad 0<t<1, \\
u(0)=u(1)=u^{\prime}(0)=u^{\prime}(1)=0, \quad D_{0^{+}}^{\beta} u(0)=0, \quad D_{0^{+}}^{\beta} u(1)=b D_{0^{+}}^{\beta} u(\eta)
\end{array}\right.
$$

is equal to

$$
\left\{\begin{array}{l}
D_{0^{+}}^{\beta} u(t)=\varphi_{q}\left(\int_{0}^{1} H(t, s) y(s) d s\right), \quad 0<t<1, \\
u(0)=u(1)=u^{\prime}(0)=u^{\prime}(1)=0 .
\end{array}\right.
$$

Lemma 2.2 ([30]) If $y \in C[0,1], B V P(2.2)$ has a unique solution

$$
u(t)=\int_{0}^{1} G(t, s) \varphi_{q}\left(\int_{0}^{1} H(s, \tau) y(\tau) d \tau\right) d s
$$

where

$$
G(t, s)=\frac{1}{\Gamma(\beta)} \begin{cases}t^{\beta-2}(1-s)^{\beta-2}[(s-t)+(\beta-2)(1-t) s], & 0 \leq t \leq s \leq 1, \\ t^{\beta-2}(1-s)^{\beta-2}[(s-t)+(\beta-2)(1-t) s]+(t-s)^{\beta-1}, & 0 \leq s \leq t \leq 1 .\end{cases}
$$

Lemma 2.3 The functions $H, G \in C([0,1] \times[0,1],[0,+\infty))$ have the following properties:

(1)

$$
H(t, s) \leq d_{1}(1-s)^{\alpha-1}, \quad t, s \in(0,1)
$$

where $d_{1}=\frac{1}{\left(1-b^{p-1} \eta^{\alpha-1}\right) \Gamma(\alpha)}$.

(2)

$$
(\beta-2) k(t) q(s) \leq \Gamma(\beta) G(t, s) \leq M_{0} q(s), \quad t, s \in(0,1),
$$

where

$$
k(t)=t^{\beta-2}(1-t)^{2}, \quad q(s)=s^{2}(1-s)^{\beta-2}, \quad M_{0}=\max \left\{\beta-1,(\beta-2)^{2}\right\} .
$$

(3)

$$
G(t, s) \geq \frac{\beta-2}{M_{0}} t^{\beta-2}(1-t)^{2} G\left(t_{0}, s\right), \quad t, s, t_{0} \in(0,1) .
$$

Proof

(1) For any $t, s \in(0,1)$,

$$
h(t, s) \leq \frac{1}{\Gamma(\alpha)}[t(1-s)]^{\alpha-1} \leq \frac{(1-s)^{\alpha-1}}{\Gamma(\alpha)},
$$

then

$$
h(\eta, s) \leq \frac{1}{\Gamma(\alpha)}[\eta(1-s)]^{\alpha-1} .
$$


Therefore

$$
\begin{aligned}
H(t, s) & \leq \frac{(1-s)^{\alpha-1}}{\Gamma(\alpha)}+\frac{b^{p-1}}{1-b^{p-1} \eta^{\alpha-1}} \frac{\eta^{\alpha-1}(1-s)^{\alpha-1}}{\Gamma(\alpha)} \\
& =\frac{(1-s)^{\alpha-1}}{\Gamma(\alpha)\left(1-b^{p-1} \eta^{\alpha-1}\right)}=d_{1}(1-s)^{\alpha-1} .
\end{aligned}
$$

(2) See Lemma 2.4(2) of [30].

(3) For any $t, s, t_{0} \in(0,1)$, we have

$$
\begin{aligned}
G(t, s) & \geq \frac{(\beta-2) k(t) q(s)}{\Gamma(\beta)} \\
& =\frac{(\beta-2) k(t)}{M_{0} \Gamma(\beta)} M_{0} q(s) \\
& \geq \frac{(\beta-2) k(t)}{M_{0} \Gamma(\beta)} \Gamma(\beta) G\left(t_{0}, s\right) \\
& =\frac{\beta-2}{M_{0}} t^{\beta-2}(1-t)^{2} G\left(t_{0}, s\right) .
\end{aligned}
$$

This completes the proof.

Remark 2.1 By Lemmas 2.2 and 2.3, if $D_{0^{+}}^{\beta} u \geq 0$ and $u(0)=u(1)=u^{\prime}(0)=u^{\prime}(1)=0$, we conclude that $u(t) \geq 0, t \in[0,1]$.

$u$ is said to be a lower solution for BVP (1.1) if $u$ satisfies the following inequality system:

$$
\left\{\begin{array}{l}
-D_{0^{+}}^{\alpha}\left(\varphi_{p}\left(D_{0^{+}}^{\beta} u(t)\right)\right) \leq f(t, u(t)), \quad 0<t<1, \\
u(0) \leq 0, \quad u(1) \leq 0, \quad u^{\prime}(0) \leq 0, \quad u^{\prime}(1) \leq 0, \\
-D_{0^{+}}^{\beta} u(0) \leq 0, \quad-D_{0^{+}}^{\beta} u(1) \leq-b D_{0^{+}}^{\beta} u(\eta) .
\end{array}\right.
$$

Similarly, we define the upper solution for BVP (1.1) by replacing "least or equal" by "greater or equal".

\section{Main result}

Theorem 3.1 Assume that the following conditions $\left(\mathrm{H}_{1}\right)-\left(\mathrm{H}_{3}\right)$ are satisfied:

$\left(\mathrm{H}_{1}\right) f(t, u) \in C((0,1) \times(0,+\infty),[0,+\infty))$ and $f(t, u)$ is nonincreasing relative to $u$.

$\left(\mathrm{H}_{2}\right)$ For any constant $\lambda>0$,

$$
0<\int_{0}^{1}(1-s)^{\alpha-1} f\left(s, \lambda s^{\beta-2}(1-s)^{2}\right) d s<+\infty .
$$

$\left(\mathrm{H}_{3}\right)$ There exist a function $a \in C[0,1]$ and a constant $k>0$ such that $a(t) \geq k t^{\beta-2}(1-t)^{2}$, $t \in[0,1]$, and

$$
\begin{aligned}
& \int_{0}^{1} G(t, r) \varphi_{q}\left(\int_{0}^{1} H(r, s) f(s, a(s)) d s\right) d r=b(t) \geq a(t), \\
& \int_{0}^{1} G(t, r) \varphi_{q}\left(\int_{0}^{1} H(r, s) f(s, b(s)) d s\right) d r \geq a(t) .
\end{aligned}
$$


Then BVP (1.1) has at least one positive solution $w$ which satisfies $w(t) \geq m t^{\beta-2} \times$ $(1-t)^{2}$ for some $m>0$.

Proof Let

$$
P=\left\{u \in C[0,1]: \text { there exists } k_{u}>0 \text { such that } u(t) \geq k_{u} t^{\beta-2}(1-t)^{2}, t \in[0,1]\right\} \text {. }
$$

Define an operator $T$ by

$$
T u(t)=\int_{0}^{1} G(t, r) \varphi_{q}\left(\int_{0}^{1} H(r, s) f(s, u(s)) d s\right) d r, \quad u \in P .
$$

For $u \in P$, there exists $k_{u}>0$ such that $u(t) \geq k_{u} t^{\beta-2}(1-t)^{2}, t \in[0,1]$. By $\left(H_{1}\right)$ and $\left(H_{2}\right)$, we have

$$
\int_{0}^{1} H(t, s) f(s, u(s)) d s \leq d_{1} \int_{0}^{1}(1-s)^{\alpha-1} f\left(s, k_{u} s^{\beta-2}(1-s)^{2}\right) d s<+\infty .
$$

Hence

$$
\begin{aligned}
\operatorname{Tu}(t) & =\int_{0}^{1} G(t, r) \varphi_{q}\left(\int_{0}^{1} H(r, s) f(s, u(s)) d s\right) d r \\
& \leq \int_{0}^{1} \frac{M_{0}}{\Gamma(\beta)} q(r) \varphi_{q}\left(\int_{0}^{1} d_{1}(1-s)^{\alpha-1} f\left(s, k_{u} s^{\beta-2}(1-s)^{2}\right) d s\right) d r \\
& =\frac{M_{0}}{\Gamma(\beta)} d_{1}^{q-1} \int_{0}^{1} q(r) d r \varphi_{q}\left(\int_{0}^{1}(1-s)^{\alpha-1} f\left(s, k_{u} s^{\beta-2}(1-s)^{2}\right) d s\right)<+\infty .
\end{aligned}
$$

On the other hand, choose $t_{0} \in(0,1)$ such that $T u\left(t_{0}\right)=k_{T_{u}}>0$. It follows from Lemma 2.3 that

$$
\begin{aligned}
T u(t) & =\int_{0}^{1} G(t, r) \varphi_{q}\left(\int_{0}^{1} H(r, s) f(s, u(s)) d s\right) d r \\
& \geq \frac{\beta-2}{\Gamma(\beta)} k(t) \int_{0}^{1} q(r) \varphi_{q}\left(\int_{0}^{1} H(r, s) f(s, u(s)) d s\right) d r \\
& \geq \frac{\beta-2}{M_{0}} k(t) T u\left(t_{0}\right)=\frac{\beta-2}{M_{0}} k_{T_{u}} t^{\beta-2}(1-t)^{2}, \quad t \in[0,1] .
\end{aligned}
$$

It follows from (3.1) and (3.2) that $T$ is well defined and $T(P) \subset P$.

Next, we determine upper and lower solutions of BVP (1.1). In fact, by simple computations, we have

$$
\begin{aligned}
& -D_{0^{+}}^{\alpha}\left(\varphi_{p}\left(D_{0^{+}}^{\beta}(T u(t))\right)\right)=f(t, u(t)), \quad t \in(0,1), \\
& \left\{\begin{array}{l}
(T u)(0)=(T u)(1)=(T u)^{\prime}(0)=(T u)^{\prime}(1)=0, \\
D_{0^{+}}^{\beta}(T u)(0)=0, \quad D_{0^{+}}^{\beta}(T u)(1)=b D_{0^{+}}^{\beta}(T u)(\eta) .
\end{array}\right.
\end{aligned}
$$

Let $b(t)=T a(t)$, then by $\left(H_{1}\right)$ and $\left(H_{3}\right)$, we have

$$
a(t) \leq T a(t)=b(t), \quad b(t)=T a(t) \geq T b(t), \quad t \in[0,1] .
$$


Since $a(t) \in P$, from (3.2), we obtain $T a(t), T b(t) \in P$. Thus, by (3.3) and (3.5),

$$
\begin{aligned}
& -D_{0^{+}}^{\alpha}\left(\varphi_{p}\left(D_{0^{+}}^{\beta}(T b)(t)\right)\right)-f(t,(T b)(t)) \leq-D_{0^{+}}^{\alpha}\left(\varphi_{p}\left(D_{0^{+}}^{\beta}(T b)(t)\right)\right)-f(t, b(t))=0 \\
& -D_{0^{+}}^{\alpha}\left(\varphi_{p}\left(D_{0^{+}}^{\beta}(T a)(t)\right)\right)-f(t,(T a)(t)) \geq-D_{0^{+}}^{\alpha}\left(\varphi_{p}\left(D_{0^{+}}^{\beta}(T a)(t)\right)\right)-f(t, a(t))=0 .
\end{aligned}
$$

Meanwhile, (3.4) implies that $T a(t), T b(t)$ satisfy the boundary conditions of BVP (1.1). Then, from (3.5) $-(3.7), \varphi(t)=T b(t)$ and $\psi(t)=T a(t)$ are lower and upper solutions of BVP (1.1), respectively.

Next, we shall show that the BVP

$$
\left\{\begin{array}{l}
-D_{0^{+}}^{\alpha}\left(\varphi_{p}\left(D_{0^{+}}^{\beta} u(t)\right)\right)=g(t, u(t)), \quad 0<t<1, \\
u(0)=u(1)=u^{\prime}(0)=u^{\prime}(1)=0, \quad D_{0^{+}}^{\beta} u(0)=0, \quad D_{0^{+}}^{\beta} u(1)=b D_{0^{+}}^{\beta} u(\eta)
\end{array}\right.
$$

has a positive solution, where

$$
g(t, u(t))= \begin{cases}f(t, \varphi(t)), & u(t)<\varphi(t) \\ f(t, u(t)), & \varphi(t) \leq u(t) \leq \psi(t) \\ f(t, \psi(t)), & u(t)>\psi(t)\end{cases}
$$

To see this, we consider the operator $A: C[0,1] \rightarrow C[0,1]$ defined as follows:

$$
A u(t)=\int_{0}^{1} G(t, r) \varphi_{q}\left(\int_{0}^{1} H(r, s) g(s, u(s)) d s\right) d r
$$

It is well known that a fixed point of the operator $A$ is a solution of BVP (3.8).

It is clear that $A$ is continuous. Since $\varphi(t) \in P$, there exists $k_{\varphi}>0$ such that $\varphi(t) \geq$ $k_{\varphi} t^{\beta-2}(1-t)^{2}, t \in[0,1]$. It follows from $\left(H_{2}\right)$ that

$$
\begin{aligned}
\int_{0}^{1} H(t, s) g(s, u(s)) d s & \leq d_{1} \int_{0}^{1}(1-s)^{\alpha-1} f(s, \varphi(s)) d s \\
& \leq d_{1} \int_{0}^{1}(1-s)^{\alpha-1} f\left(s, k_{\varphi} s^{\beta-2}(1-s)^{2}\right) d s<+\infty
\end{aligned}
$$

Consequently, for $u \in C[0,1]$ and $t \in[0,1]$, by (3.9) and (3.10), we have

$$
\begin{aligned}
A u(t) & =\int_{0}^{1} G(t, r) \varphi_{q}\left(\int_{0}^{1} H(r, s) g(s, u(s)) d s\right) d r \\
& \leq M_{0} \int_{0}^{1} q(r) \varphi_{q}\left(\int_{0}^{1} d_{1}(1-s)^{\alpha-1} g(s, u(s)) d s\right) d r \\
& \leq M_{0} d_{1}^{q-1} \int_{0}^{1} q(r) d r \varphi_{q}\left(\int_{0}^{1}(1-s)^{\alpha-1} f\left(s, k_{\varphi} s^{\beta-2}(1-s)^{2}\right) d s\right)<+\infty
\end{aligned}
$$

which implies that $A$ is uniformly bounded. 
On the other hand, since $G(t, s)$ is continuous on $[0,1] \times[0,1]$, it is uniformly continuous on $[0,1] \times[0,1]$. Thus, for $s \in[0,1]$ and for each $\varepsilon>0$, there exists $\delta>0$ such that $\left|t_{1}-t_{2}\right|<\delta$ implies

$$
\left|G\left(t_{1}, s\right)-G\left(t_{2}, s\right)\right|<\frac{\varepsilon}{\varphi_{q}\left(d_{1} \int_{0}^{1}(1-s)^{\alpha-1} f\left(s, k_{\varphi} s^{\beta-2}(1-s)^{2}\right) d s\right)} .
$$

Furthermore, for $u \in C[0,1]$,

$$
\begin{aligned}
& \left|A u\left(t_{1}\right)-A u\left(t_{2}\right)\right| \\
& \left.\quad \leq \int_{0}^{1} \mid G\left(t_{1}, r\right)-G\left(t_{2}, r\right)\right) \mid \varphi_{q}\left(\int_{0}^{1} H(r, s) g(s, u(s)) d s\right) d r \\
& \quad \leq \int_{0}^{1}\left|G\left(t_{1}, r\right)-G\left(t_{2}, r\right)\right| \varphi_{q}\left(\int_{0}^{1} d_{1}(1-s)^{\alpha-1} f(s, \varphi(s)) d s\right) d r \\
& \quad \leq \int_{0}^{1}\left|G\left(t_{1}, r\right)-G\left(t_{2}, r\right)\right| d r \varphi_{q}\left(d_{1} \int_{0}^{1}(1-s)^{\alpha-1} f\left(s, k_{\varphi} s^{\beta-2}(1-s)^{2}\right) d s\right)<\varepsilon,
\end{aligned}
$$

which implies that $A$ is equicontinuous. Thus, the Ascoli-Arzela theorem guarantees $A$ is a compact operator. It follows from Schauder's fixed point theorem that $A$ has a fixed point $w$, i.e., $w=A w$. Consequently, (3.8) has a solution.

Finally, we will show that BVP (1.1) has at least one positive solution. In fact, we only need to prove that $\varphi(t) \leq w(t) \leq \psi(t), t \in[0,1]$. By $\left(H_{1}\right)$, we have

$$
f(t, \psi(t)) \leq g(t, w(t)) \leq f(t, \varphi(t)), \quad t \in[0,1] .
$$

It follows from (3.5) and $\left(H_{3}\right)$ that

$$
f(t, b(t)) \leq g(t, w(t)) \leq f(t, a(t)), \quad t \in[0,1] .
$$

Since $a(t) \in P$, by (3.3), we have

$$
-D_{0^{+}}^{\alpha}\left(\varphi_{p}\left(D_{0^{+}}^{\beta} \psi(t)\right)\right)=-D_{0^{+}}^{\alpha}\left(\varphi_{p}\left(D_{0^{+}}^{\beta}(T a)(t)\right)\right)=f(t, a(t)), \quad t \in(0,1) .
$$

By (3.4), (3.5), (3.11), and (3.12), we have

$$
\begin{aligned}
& -D_{0^{+}}^{\alpha}\left(\varphi_{p}\left(D_{0^{+}}^{\beta} \psi(t)\right)\right)-\left[-D_{0^{+}}^{\alpha}\left(\varphi_{p}\left(D_{0^{+}}^{\beta} w(t)\right)\right)\right]=f(t, a(t))-g(t, w(t)) \geq 0, \quad t \in[0,1], \\
& (\psi-w)(0)=(\psi-w)(1)=(\psi-w)^{\prime}(0)=(\psi-w)^{\prime}(1)=0 \\
& D_{0^{+}}^{\beta}(\psi-w)(0)=0, \quad D_{0^{+}}^{\beta}(\psi-w)(1)=b D_{0^{+}}^{\beta}(\psi-w)(\eta)
\end{aligned}
$$

Setting $z=\varphi_{p}\left(D_{0^{+}}^{\beta} \psi(t)\right)-\varphi_{p}\left(D_{0^{+}}^{\beta} w(t)\right)$, then

$$
\begin{aligned}
& -D_{0^{+}}^{\alpha} z(t)=-D_{0^{+}}^{\alpha}\left(\varphi_{p}\left(D_{0^{+}}^{\beta} \psi(t)\right)\right)-\left[-D_{0^{+}}^{\alpha}\left(\varphi_{p}\left(D_{0^{+}}^{\beta} w(t)\right)\right)\right] \geq 0, \\
& z(0)=0, \quad z(1)=\varphi_{p}(b) z(\eta) .
\end{aligned}
$$

Hence, by Lemma 2.1, we get $z(t) \geq 0, t \in[0,1]$. Since $\varphi_{p}$ is monotone increasing, we have $D_{0^{+}}^{\beta} \psi(t) \geq D_{0^{+}}^{\beta} w(t)$, that is, $D_{0^{+}}^{\beta}(\psi(t)-w(t)) \geq 0, t \in[0,1]$. By Remark 2.1, we have $w(t) \leq$ 
$\psi(t)$ for $t \in[0,1]$. Similarly, $w(t) \geq \varphi(t)$ on $[0,1]$. Therefore, $w(t)$ is a positive solution of $\operatorname{BVP}(1.1)$. And $\varphi(t) \in P$ implies that there exists $m>0$ such that $w(t) \geq \varphi(t) \geq m t^{\beta-2}(1-$ $t)^{2}, t \in[0,1]$. This completes the proof.

\section{An example}

Example 4.1 Consider the following fractional singular BVP:

$$
\begin{cases}-D_{0^{+}}^{\frac{3}{2}}\left(\varphi_{p}\left(D_{0^{+}}^{\frac{7}{2}} u(t)\right)\right)=(1-t)^{-\frac{1}{4}} u^{-\frac{1}{2}}, & 0<t<1, \\ u(0)=u(1)=u^{\prime}(0)=u^{\prime}(1)=0, & D_{0^{+}}^{\frac{7}{2}} u(0)=0, \quad D_{0^{+}}^{\frac{7}{2}} u(1)=\frac{1}{4} D_{0^{+}}^{\frac{7}{2}} u\left(\frac{1}{2}\right),\end{cases}
$$

where $\varphi_{p}(t)=|t|^{p-2} t, p>1$. Then BVP (4.1) has a positive solution $w(t) \geq m t^{\frac{3}{2}}(1-t)^{2}$ for some $m>0$.

In fact, let $\alpha=\frac{3}{2}, \beta=\frac{7}{2}, f(t, u)=(1-t)^{-\frac{1}{4}} u^{-\frac{1}{2}}, t \in(0,1)$. Obviously, $f(t, u)$ is singular at $t=1$ and $u=0$. It is easy to check that $\left(H_{1}\right)$ in Theorem 3.1 is satisfied. For any constant $\lambda>0$,

$$
\begin{aligned}
0 & <\int_{0}^{1}(1-s)^{\alpha-1} f\left(s, \lambda s^{\beta-2}(1-s)^{2}\right) d s \\
& =\int_{0}^{1}(1-s)^{\frac{1}{2}}(1-s)^{-\frac{1}{4}}\left[\lambda s^{\frac{3}{2}}(1-s)^{2}\right]^{-\frac{1}{2}} d s \\
& =\lambda^{-\frac{1}{2}} \int_{0}^{1} s^{-\frac{3}{4}}(1-s)^{-\frac{3}{4}} d s \\
& =\lambda^{-\frac{1}{2}} B\left(\frac{1}{4}, \frac{1}{4}\right)=\lambda^{-\frac{1}{2}} \frac{\Gamma^{2}\left(\frac{1}{4}\right)}{\sqrt{\pi}}<+\infty,
\end{aligned}
$$

so $\left(H_{2}\right)$ in Theorem 3.1 is satisfied.

Set $\mu=\frac{1}{2}$, then $f(t, u) \leq f(t, r u) \leq r^{-\mu} f(t, u)$ for any $r \in(0,1)$. Since $e(t)=t^{\frac{3}{2}}(1-t)^{2} \in P$, by (3.2) we know $T e \in P, T^{2} e \in P$, then there exist positive numbers $k$ and $l$ such that $T e \geq k e$ and $T^{2} e \geq l e$. Take $0<r_{0}<\min \left\{1, k, l^{\frac{1}{1-\mu^{2}}}\right\}$, then

$$
T\left(r_{0} e\right) \geq T e \geq k e \geq r_{0} e, \quad T^{2}\left(r_{0} e\right) \geq r_{0}^{\mu^{2}} T^{2} e \geq r_{0}^{\mu^{2}} l e \geq r_{0} e .
$$

If we take $a(t)=r_{0} t^{\frac{3}{2}}(1-t)^{2}$, then condition $\left(H_{3}\right)$ of Theorem 3.1 is satisfied. Consequently, the above conclusion is guaranteed by Theorem 3.1.

\section{Conclusion}

In this paper, we consider the fractional singular three-point boundary value problem with $p$-Laplacian operator. It is worth pointing out that $f(t, u)$ may be singular at $t=0,1$ and $u=0$. Some properties of the associated Green function are obtained. By using the upper and lower solutions method and a fixed point theorem, the existence result of positive solution is established. 
List of abbreviations

Not applicable.

\section{Availability of data and materials}

Not applicable.

\section{Competing interests}

The authors declare that they have no competing interests.

\section{Authors' contributions}

All authors contributed equally to the writing of this paper. The authors read and approved the final manuscript.

\section{Publisher's Note}

Springer Nature remains neutral with regard to jurisdictional claims in published maps and institutional affiliations.

Received: 2 January 2018 Accepted: 2 April 2018 Published online: 10 April 2018

\section{References}

1. Chen, T., Liu, W., Hu, Z:: A boundary value problem for fractional differential equation with $p$-Laplacian operator at resonance. Nonlinear Anal. 75, 3210-3217 (2012)

2. Tan, J., Cheng, C.: Existence of solutions of boundary value problems for fractional differential equations with p-Laplacian operator in Banach spaces. Numer. Funct. Anal. Optim. 38, 738-753 (2017)

3. Shen, T., Liu, W., Shen, X.: Existence and uniqueness of solutions for several BVPs of fractional differential equations with $p$-Laplacian operator. Mediterr. J. Math. 13, 4623-4637 (2016)

4. Dong, X., Bai, Z., Zhang, S.: Positive solutions to boundary value problems of $p$-Laplacian with fractional derivative. Bound. Value Probl. 2017, 5 (2017)

5. Liang, S., Zhang, J.: Existence and uniqueness of positive solutions for integral boundary problems of nonlinear fractional differential equations with p-Laplacian operator. Rocky Mt. J. Math. 44, 953-974 (2014)

6. Liu, Z., Lu, L.: A class of BVPs for nonlinear fractional differential equations with $p$-Laplacian operator. Electron. J. Qual. Theory Differ. Equ. 2012, 70 (2012)

7. Hao, X., Wang, H., Liu, L., Cui, Y.: Positive solutions for a system of nonlinear fractional nonlocal boundary value problems with parameters and p-Laplacian operator. Bound. Value Probl. 2017, 182 (2017)

8. Jafari, H., Baleanu, D., Khan, H., Khan, R.A., Khan, A.: Existence criterion for the solutions of fractional order $p$-Laplacian boundary value problems. Bound. Value Probl. 2015, 164 (2015)

9. Guo, X.: Existence of unique solution to switched fractional differential equations with $p$-Laplacian operator. Turk. J. Math. 39, 864-871 (2015)

10. Ding, Y., Wei, Z., Xu, J., O'Regan, D.: Extremal solutions for nonlinear fractional boundary value problems with p-Laplacian. J. Comput. Appl. Math. 288, 151-158 (2015)

11. $\mathrm{Xu}, \mathrm{J}$., Dong, W.: Existence and uniqueness of positive solutions for a fractional boundary value problem with p-Laplacian operator. Acta Math. Sin. (Chin. Ser.) 59, 385-396 (2016)

12. Liu, X., Jia, M., Ge, W.: The method of lower and upper solutions for mixed fractional four-point boundary value problem with p-Laplacian operator. Appl. Math. Lett. 65, 56-62 (2017)

13. Wang, J., Xiang, H.: Upper and lower solutions method for a class of singular fractional boundary value problems with p-Laplacian operator. Abstr. Appl. Anal. 2010, Article ID 971824 (2010)

14. Wang, Y., Liu, L., Wu, Y.: Extremal solutions for $p$-Laplacian fractional integro-differential equation with integral conditions on infinite intervals via iterative computation. Adv. Differ. Equ. 2015, 24 (2015)

15. Wang, Y., Jiang, J.: Existence and nonexistence of positive solutions for the fractional coupled system involving generalized $p$-Laplacian. Adv. Differ. Equ. 2017, 337 (2017)

16. Li, S., Zhang, X., Wu, Y., Caccetta, L.: Extremal solutions for $p$-Laplacian differential systems via iterative computation. Appl. Math. Lett. 26, 1151-1158 (2013)

17. Zhang, X., Liu, L., Wiwatanapataphee, B., Wu, Y.: The eigenvalue for a class of singular $p$-Laplacian fractional differential equations involving the Riemann-Stieltjes integral boundary condition. Appl. Math. Comput. 235, 412-422 (2014)

18. Ren, T., Li, S., Zhang, X., Liu, L.: Maximum and minimum solutions for a nonlocal $p$-Laplacian fractional differential system from eco-economical processes. Bound. Value Probl. 2017, 118 (2017)

19. Wang, Y., Liu, L., Wu, Y.: Positive solutions for a nonlocal fractional differential equation. Nonlinear Anal. 74, 3599-3605 (2011)

20. Zhang, X.: Positive solutions for a class of singular fractional differential equation with infinite point boundary value conditions. Appl. Math. Lett. 39, 22-27 (2015)

21. Hao, X., Liu, L., Wu, Y.: Positive solutions for nonlinear fractional semipositone differential equation with nonlocal boundary conditions. J. Nonlinear Sci. Appl. 9, 3992-4002 (2016)

22. Hao, X.: Positive solution for singular fractional differential equations involving derivatives. Adv. Differ. Equ. 2016, 139 (2016)

23. Zhang, X., Zhong, Q.: Uniqueness of solution for higher-order fractional differential equations with conjugate type integral conditions. Fract. Calc. Appl. Anal. 20, 1471-1484 (2017)

24. Zhang, X., Zhong, Q.: Triple positive solutions for nonlocal fractional differential equations with singularities both on time and space variable. Appl. Math. Lett. 80, 12-19 (2018)

25. Zou, Y., He, G.: On the uniqueness of solutions for a class of fractional differential equations. Appl. Math. Lett. 74 68-73 (2017)

26. Cui, Y.: Uniqueness of solution for boundary value problems for fractional differential equations. Appl. Math. Lett. 51, 48-54 (2016) 
27. Zou, Y., Cui, Y.: Existence results for a functional boundary value problem of fractional differential equations. Adv. Differ. Equ. 2013, 233 (2013)

28. Pu, R., Zhang, X., Cui, Y., Li, P., Wang, W.: Positive solutions for singular semipositone fractional differential equation subject to multipoint boundary conditions. J. Funct. Spaces 2017, Article ID 5892616 (2017)

29. Podlubny, l.: Fractional Differential Equations. Academic Press, New York (1999)

30. Xu, X., Jiang, D., Yuan, C.: Multiple positive solutions for the boundary value problem of a nonlinear fractional differential equation. Nonlinear Anal. 71, 4676-4688 (2009)

Submit your manuscript to a SpringerOpen ${ }^{\circ}$ journal and benefit from:

- Convenient online submission

- Rigorous peer review

- Open access: articles freely available online

- High visibility within the field

- Retaining the copyright to your article

Submit your next manuscript at $\gg$ springeropen.com 